\title{
EU-Richtlinie erfordert „verletzungssichere“ Spritzen
}

\author{
Für die Umsetzung der EU-Richtlinie 2010/32/EU zur Vermeidung von \\ Verletzungen durch scharfe bzw. spitze Instrumente in nationales Recht \\ galt eine Frist bis Mai dieses Jahres. Von den sich daraus ergebenden An- \\ forderungen für die Praxis ist im HNO-Bereich insbesondere auch die \\ subkutane Immuntherapie (SCIT) betroffen.
}

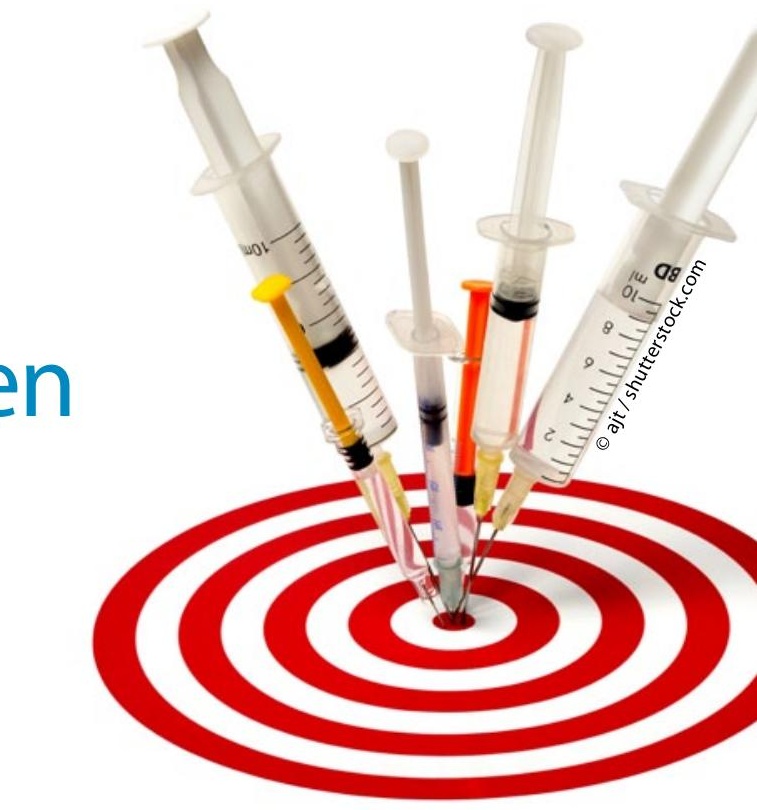

mit einem Finger über die Kanüle geklappt wird und dort einrastet, sodass Gesundheitspersonal vor Stichverletzungen mit der kontaminierten Spitze geschützt wird.

Auch für subkutane Injektionen einer allergenspezifischen Immuntherapie gemäß den Leitlinien des Ärzteverbands Deutscher Allergologen und der Deutschen Gesellschaft für Allergologie und klinische Immunologie [abrufbar unter www.awmf.org] gibt es verletzungssichere Injektionsnadeln/Kanülensysteme, die den dort geforderten Spezifikationen entsprechen.

Ein großer Allergenhersteller hat bereits auf die rechtlichen Veränderungen ab Mai reagiert und liefert seine Präparate zur subkutanen spezifischen Immuntherapie zukünftig mit verletzungssicheren Injektionsnadeln aus. Es handelt sich hierbei um Einmalspritzen mit einem fest mit der Kanüle verbundenen Schutzschild, das nach der Injektion mit einem Finger über die Kanüle geklappt wird.

Da davon ausgegangen werden kann, dass die Gesundheitsämter und andere behördliche Stellen, die in den einzelnen Regierungspräsidien für die Einhaltung der Biostoffverordnung zuständig sind. ab Mai verstärkt die Einhaltung dieser Vorgaben überprüfen werden, sei allen HNO-Ärzten empfohlenn, sich aktiv dieser Problematik anzunehmen.

Prof. Dr. med. Ludger Klimek

Zentrum für Rhinologie und Allergologie

An den Quellen 10

D-65183 Wiesbaden

E-Mail: ludger.klimek@allergiezentrum.org 